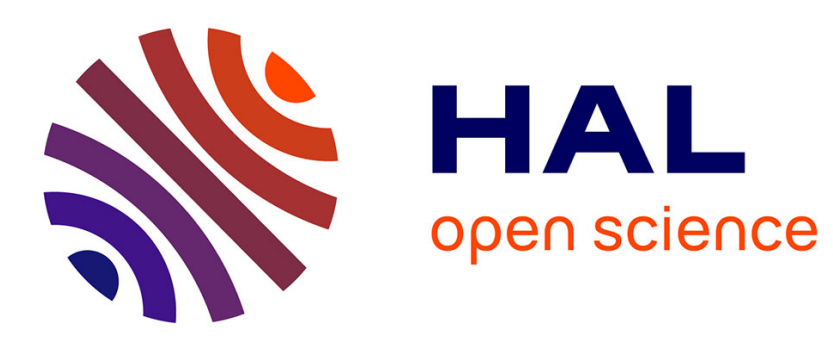

\title{
Time-resolved torsional relaxation of spider draglines by an optical technique.
}

Olivier Emile, Albert Le Floch, F. Vollrath

\section{To cite this version:}

Olivier Emile, Albert Le Floch, F. Vollrath. Time-resolved torsional relaxation of spider draglines by an optical technique.. Physical Review Letters, 2007, 98 (16), pp.167402. 10.1103/PhysRevLett.98.167402 . hal-00908328

\section{HAL Id: hal-00908328 \\ https://hal.science/hal-00908328}

Submitted on 22 Nov 2013

HAL is a multi-disciplinary open access archive for the deposit and dissemination of scientific research documents, whether they are published or not. The documents may come from teaching and research institutions in France or abroad, or from public or private research centers.
L'archive ouverte pluridisciplinaire HAL, est destinée au dépôt et à la diffusion de documents scientifiques de niveau recherche, publiés ou non, émanant des établissements d'enseignement et de recherche français ou étrangers, des laboratoires publics ou privés. 


\title{
Time-Resolved Torsional Relaxation of Spider Draglines by an Optical Technique
}

\author{
O. Emile, ${ }^{1, *}$ A. Le Floch, ${ }^{1}$ and F. Vollrath ${ }^{2}$ \\ ${ }^{1}$ Laboratoire d'Electronique Quantique-Physique des Lasers, UMR CNRS 6627, Université de Rennes I, 35042 Rennes Cedex, France \\ ${ }^{2}$ Department of Zoology, South Parks Road, Oxford OX1 3PS, United Kingdom
}

(Received 12 October 2006; published 18 April 2007)

\begin{abstract}
The sensitivity of the torsional pendulum demonstrates the self-shape-memory effect in different types of spider draglines. Here we report the time-resolved noncovalent bonds recovery in the protein structure. The torsional dynamics of such multilevel structure governed by reversible interactions are described in the frame of a nested model. Measurement of three different relaxation times confirms the existence of three energy storage levels in such two protein spidroin systems. Torsion opens the way to further investigations towards unraveling the tiny torque effects in biological molecules.
\end{abstract}

DOI: 10.1103/PhysRevLett.98.167402

Silk fibers and spider draglines have attracted much interest because of their unusual mechanical properties including high strength and toughness [1-4]. Those properties remain even at cryogenic temperatures, making spider silks the perfect threads to hold in place the small targets in inertial confinement laser fusion experiments [5]. The supramolecular structures of spider silk proteins represent a unique system at the intersection of material science, biophysics, and molecular biology. Although much progress has been made towards understanding the underlying principles of their mechanical properties [6], much remains to be done. Specifically, it appears that an analysis of torsional properties might open a window to new insights [7]. Spider dragline silk is a composite material with a multilevel structure which amino-acid sequences have been optimized over millions of years of biological evolution [8]. They are mainly composed of two proteins organized in oriented small $\beta$-sheet crystals and less crystalline domains [9]. The ultimate goal in the field of protein based materials has been the ability to duplicate spider draglines properties "in vitro" to develop novel biomaterials. In recent years, significant progresses have been made both in isolating spiderlike proteins and in silk spinning [10,11] with much success in the first field (protein analysis) but little in the second field (protein assembly).

The torsional properties of silk might allow us to bridge the gap between the two approaches. For two centuries, torsion pendulums have been used to measure weak forces between fundamental electrostatic [12] and gravity interactions [13]. Today they are still the most sensitive tools to test the gravitational inverse square law at length scales below $1 \mathrm{~mm}$ [14-16]. One may wonder whether the sensitivity of the torsion pendulum could also permit to explore the dynamics of the relaxation of the amino-acid polymers of the spider draglines. It is the aim of this Letter to explore their torsional properties within the framework of a nested model, taking into account the different levels of complexity of these molecular and supramolecular structures.

First we demonstrate the existence of the torsional selfshape-memory effect in different types of spider draglines, a thread that exhibits a behavior different not only from
PACS numbers: 78.20.Hp, 78.40.Me, 87.10.+e, 87.15.-v

man-made polymers or crystalline metallic threads but also from the common visco-elastic filaments. Our experimental setup (Fig. 1) employed a small plastic or copper rod mimicking the spider weight and suspended from a $10-\mathrm{cm}-$ long sample thread. Great care is taken to isolate the thread from any external perturbations. The "spider rod" is then carefully excited to achieve a twist angle of about $50^{\circ}$. The dynamical responses of the different systems are registered via a camera attached to a computer.

The double-strand spider dragline from an Araneus diadematus has a diameter of $3 \mu \mathrm{m}$, measured with an electron microscope (see inset of Fig. 1). Away from its original position, it weakly oscillates [see Fig. 2(a)] around a pseudo equilibrium position as can be seen in the inset, which is a zoom of the self-relaxation dynamics. During the excitation regime, i.e., up to the red arrow of Fig. 2(a), one may wonder whether part of the energy has been dissipated within the thread. However, and most intriguingly, the spider thread slowly self-recovers its original

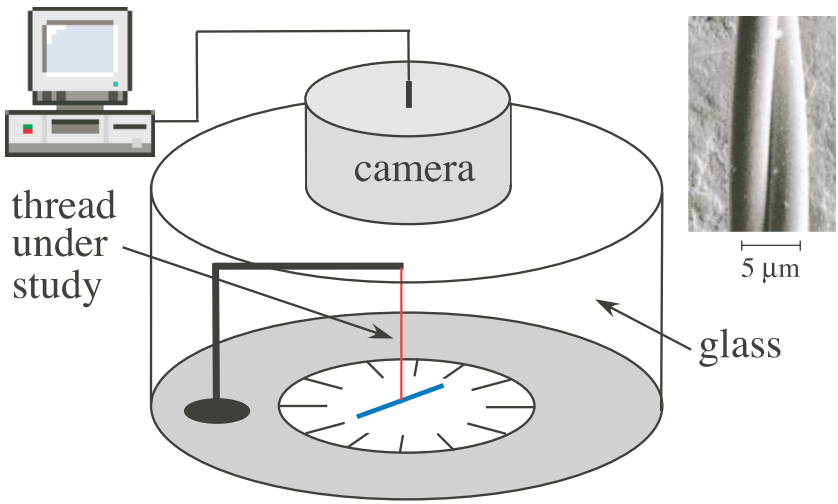

FIG. 1 (color). Experimental setup. A rod made of plastic or copper, mimicking the spider, is suspended to a 10 -cm-long spider dragline or man-made threads in a torsion pendulum experiment. The whole experiment is settled on an antivibration honeycomb core optical table. Hydrometry: $(40 \pm 1) \%$, temperature: $(20.0 \pm 0.1)^{\circ} \mathrm{C}$. Inset: photograph of a typical goldcoated spider dragline taken with a scanning electronic microscope (Cambridge Instruments). 


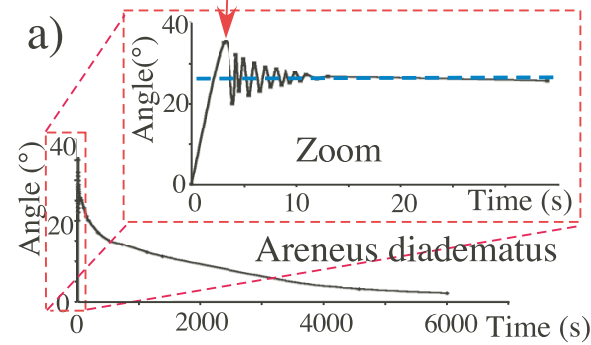

b)

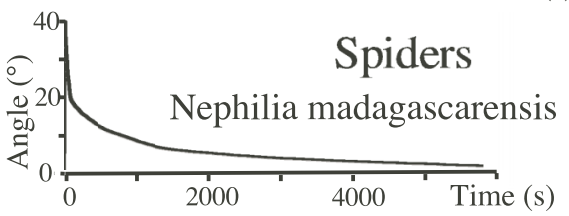

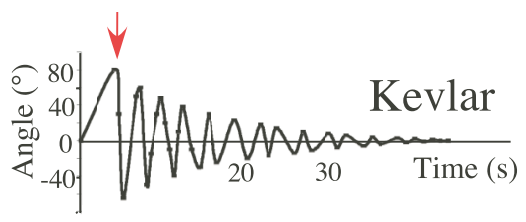

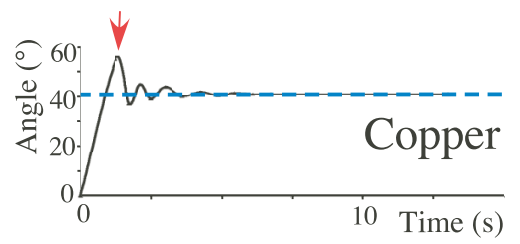

FIG. 2 (color). Experimental torsion pendulum relaxation dynamics of four threads. Red arrows: end of the applied torque and beginning of the free relaxation period. (a) Araneus diadematus (suspended mass $0.1 \mathrm{~g}$ ) and zoom of the beginning of the relaxation dynamics (inset) and Nephila (suspended mass $0.5 \mathrm{~g}$ ) spider threads. (b) $10 \mu \mathrm{m}$-diameter polyparaphenylene terephthalamide (Kevlar 29) thread (suspended mass $0.5 \mathrm{~g}$, torsional constant $C=10^{-9} \mathrm{Nm} / \mathrm{rad}$ ) and $50 \mu \mathrm{m}$-diameter copper thread. Blue dotted line: new equilibrium position. position in an apparently exponential manner. This response differs from the behavior of man-made threads, but is common to different types of dragline threads. We have also observed such behavior on Pholcus phalangioides, Zygiella x-notata, and Nephila madagascarensis. The response of this last spider dragline, where the diameter reaches $6 \mu \mathrm{m}$, exhibits the same low resilience behavior, as displayed in Fig. 2(a).

Let us compare the spider dragline experiment with that using a tough $10 \mu \mathrm{m}$-thick Kevlar ${ }^{\mathrm{TM}}$ thread. After excitation from its initial equilibrium angular position, the rod oscillates gently around its original position [see Fig. 2(b)]. For this man-made polymer, the response was elastic. This is the typical weakly damped, torsional pendulum oscillating behavior. The rather soft $50 \mu \mathrm{m}$-diameter metallic copper thread had a much "improved" torsional stability as, twisted from its original rest position, the rod barely oscillated around a new-deformed-equilibrium position [Fig. 2(b)]. Thus, the copper thread, unlike the Kelvar thread, showed low resilience linked to high-energy dissipation. Hence this thread becomes hard and inelastic since the deformation is due to the appearance of microscopic incoherent dislocations, i.e., imperfections in the crystal. Such dislocations are stuck by the impurities in the crystal structure and interfere with one another as described in the Bragg-Nye crystal model [17]. The copper thread has been work hardened and cannot be easily untwist again. Actually, copper is known to become brittle after a few uses [18]. Its mechanical properties lead to a high torsional stability but at the cost of the thread losing its original softness properties. Moreover, the twist deformations are irreversible even if the temperature is raised. Neither the oscillating Kevlar thread nor the nonoscillating copper thread matches the functional response of the spider thread.

This torsional behavior is also different from that of common visco-elastic threads. For pulsed excitation, a commercially available rubber resin adhesive system (RRAS) exhibits a quasielastic response [Fig. 3(a)]. However, if we apply a constant excitation for a $20 \mathrm{~s}$ period, the system relaxes towards a new equilibrium position but never recovers its initial position. In this case, the viscoelastic creep is the main mechanism and the inset shows the only partial exponential creep recovery that we analyze below. None of these regimes are suited for the spider which needs a tough thread which hardly twists and that slowly recovers its initial position if spuriously excited. Such a mechanical response might serve to protect the spider from visually hunting predators. In its behavior, the spider dragline can be compared with a man-made shape-memory nickel-titanium (nitinol) alloy thread widely used in industry [19]. Like the spider dragline thread, once excited, the nitinol thread weakly oscillated around a new equilibrium position that is different from its original one [Fig. 3(b)]. This behavior resembles that of the copper thread but, as shown in Fig. 3(b), when heated with an external stimulus, the nitinol thread recovered its original shape. The mechanisms underlying the equilibrium positions differ between the ductile copper and the shape-memory nitinol threads. There are no spurious dislocations in nitinol, allowing several twists without the thread breaking. Its properties are due to a diffusionless coherent martensitic nucleation $[20,21]$ leading to the de-
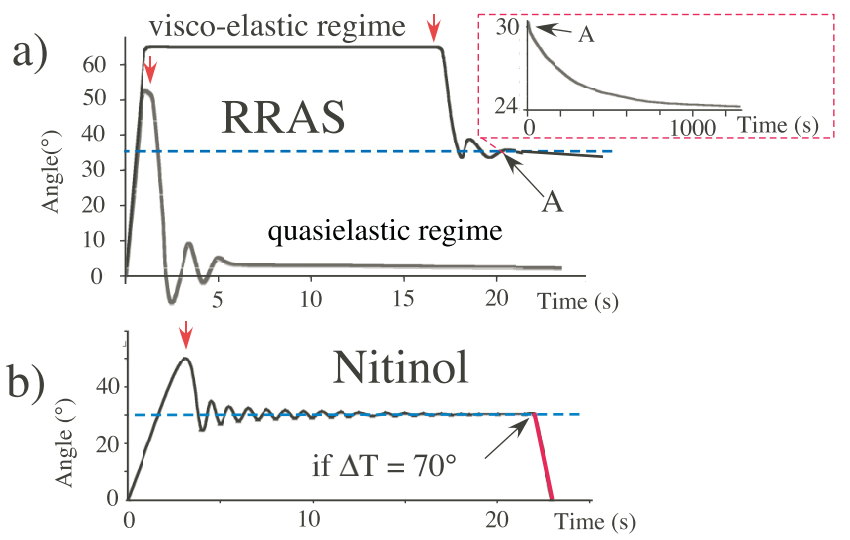

FIG. 3 (color). Experimental torsional relaxation dynamics of (a) a RRAS both in pulsed excitation regime and in constant excitation regime, (b) a $100 \mu \mathrm{m}$-diameter shape-memory nitinol alloy thread (Dynalloy, Inc.). To relax to its original shape, the alloy needs to be heated to $90{ }^{\circ} \mathrm{C}$. 
formation of the crystal structure with low energy dissipation. However, whereas the recovery of shape and position is a self-reform mechanism in the case of the spider dragline, the reversibility of the nitinol deformation requires exposure to an external stimulus (such as heating to $90^{\circ} \mathrm{C}$ ). Recently, in a pioneering experiment, shape memory has been discovered in polymers containing cinnamic groups [22] when exposed to light at a given wavelength. It appears that in the spider dragline threads, selection against twisting has led to the evolution of a self-shape-memory material.

We interpret the self-repair in spider's silk filament to be based on reformations of noncovalent bonds that mainly include hydrogen bonds [23] and van der Waals interactions. These would occur at different levels of the complex multilevel structure [24-26] of a dragline silk. The socalled secondary structure, for instance, a helix, represents the conformation of the primary amino-acid sequences. All domains in the dragline silk have a preferred secondary structure and are strongly oriented with the chains parallel to the fiber. The majority of the alanine residues are incorporated in regular $\beta$ sheets that form the microcrystalline domains and that also include part of the glycine residues. The remaining glycine rich part forms the surrounding helical structures in a less crystalline matrix [9]. These secondary structures are mainly maintained by rather strong and highly directional hydrogen bonds. Structures in silk, beyond the secondary structure, include a larger tertiary structure with different folding patterns in the presence of hydrogen and van der Waals bonds (see inset of Fig. 4). A quaternary fibrillar organization in the silk includes the two main proteins organized supramolecular helices and $\beta$ sheet where van der Waals interactions are ubiquitous.

Such multilevel structures could help us to model the torsion and the relaxation of the spider dragline in the frame of a nested model. Let us consider a small portion of a spider dragline. The portion length could be of the order of the typical size of an amino-acid sequence, i.e., of the order of $10 \mathrm{~nm}$. When a global torque is applied to the thread, every portion is submitted to a small torque $\Gamma$. The relaxation of the thread behaves in an exponential fashion [Fig. 2(a)], which suggests that the applied torque varies exponentially with the twist angle on each portion in the following form:

$$
\Gamma \propto \exp \left(\theta / \Theta_{0}\right),
$$

where $\Theta_{0}$ is a constant. Each section of the dragline thread, whose diameter is of the order of $3 \mu \mathrm{m}$, contains several juxtapositions of quaternary structures. Depending on the torque, the twist could be decomposed in two parts $\theta(\Gamma)=$ $\theta_{4}(\Gamma)+\Delta \theta_{n<4}(\Gamma) . \theta_{4}(\Gamma)$ accounts for the twist due to the modification of the bonds within the quaternary structure itself whereas $\Delta \theta_{n<4}(\Gamma)$ accounts for the twist angle variation due to the inner lower structures. Let us denote $n_{3}$ the number of tertiary structure within one quaternary structure. Every twist in the tertiary structure can be again

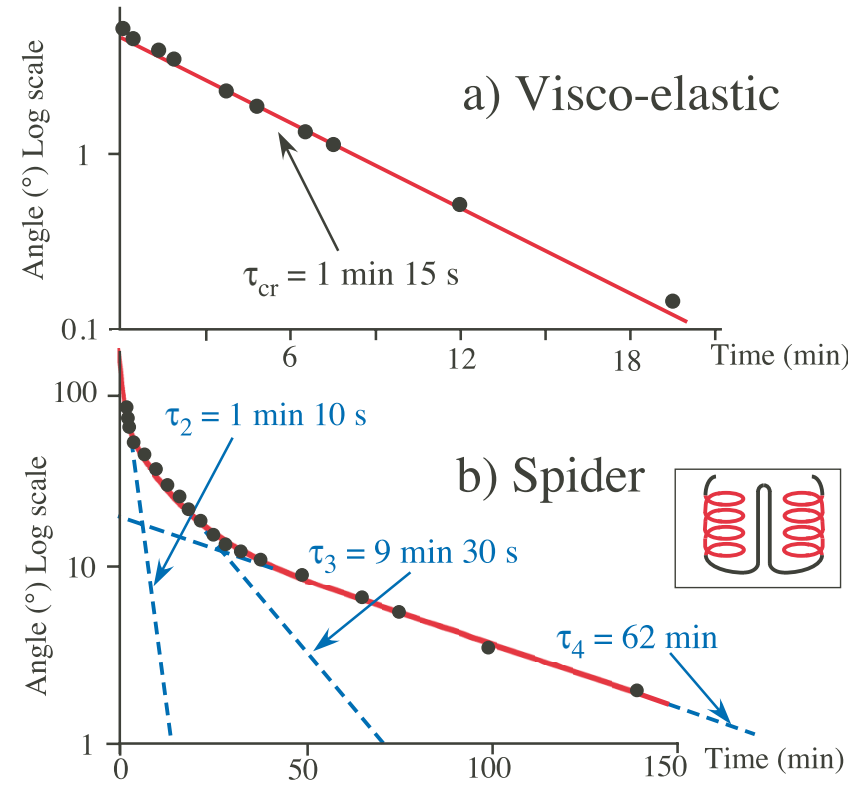

FIG. 4 (color). Torsional logarithm relaxation behavior of (a) the RRAS of Fig. 3(a), (b) the Araneus diadematus spider dragline of Fig. 2(a). Black points: experimental measurements, red line: theoretical fit with a function of the form of Eq. (3), blue dotted straight lines: time constants associated with the different torsional levels. Inset: an example of a tertiary protein structure composed of secondary structures (here $\alpha$ helices in red). The quaternary structure involves more than one amino-acid chain. Such nm-scale proteins are present in one section of a spider thread.

decomposed in one part $\theta_{3}(\Gamma)$ accounting for the modification of the bonds within the tertiary structure and an other part $\theta_{n<3}(\Gamma)$ accounting for the twist angle variation due to the rotation of the lower structures. Then one must have $\Delta \theta_{n<4}(\Gamma)=n_{3}\left[\theta_{3}(\Gamma)+\Delta \theta_{n<3}(\Gamma)\right]$, where $\Delta \theta_{n<3}(\Gamma)$ can also be decomposed. One finally gets:

$$
\theta(\Gamma)=\theta_{4}(\Gamma)+n_{3}\left\{\theta_{3}(\Gamma)+n_{2}\left[\theta_{2}(\Gamma)+\ldots \ldots\right]\right\} .
$$

Such an expression describes the steady state solution of the torsional differential equation for the twist angle. This holds for a given torque for a section of the spider thread in the frame of the nested model. It can be summed to obtain the total angle variation.

Besides, in the case of torsion thanks to gravity, the spider itself can give access to the dynamics of the relaxation during the self-recovery period. Depending on the level of the structure, the relaxation time constants should be different, the higher structure having the largest time constant. Thus the relaxation of the spider dragline, which is the solution of the free torsional equation, should read:

$$
\theta(t)=\theta_{2} \exp \left(-t / \tau_{2}\right)+\theta_{3} \exp \left(-t / \tau_{3}\right)+\theta_{4} \exp \left(-t / \tau_{4}\right),
$$

where $\tau_{2}, \tau_{3}, \tau_{4}$ are the time constants associated with the different levels, for a given experiment. In case of Araneus 
diadematus dragline, the sensitivity of the torsion pendulum gives a direct experimental access to three different time constants as can be seen on the logarithmic scale relaxation dynamics of Fig. 4(b). The theoretical adjustment from Eq. (3) leads to $\tau_{2}=1 \min 10 \mathrm{~s} \pm 5 \mathrm{~s}, \tau_{3}=$ $9 \min 30 \mathrm{~s} \pm 30 \mathrm{~s}$, and $\tau_{4}=62 \mathrm{~min} \pm 4 \mathrm{~min}$ in the spiderlike regime. The almost 1 order of magnitude observed between the three relaxation times can be justified by the increasing dimensions associated with each level of the whole structure and the relative values of the different interactions. Although the diameter of the Nephila madagascarensis thread is 2 times larger than that of the Araneus spider, we observe also three time constants $\left(\tau_{2}=\right.$ $2 \mathrm{~min} 10 \mathrm{~s}, \tau_{3}=15 \mathrm{~min} 50 \mathrm{~s}$, and $\tau_{4}=85 \mathrm{~min}$ ) with similar ratios for a "spider rod" of $0.5 \mathrm{~g}$. Note that such three time constant response in draglines is typical of these two fibroin systems. We have checked that similar relaxations are observed in a single strand thread as well as in two and four assembled threads. This is qualitatively different from that of a visco-elastic thread which is associated with the creep recovery and can be described as: $\theta(t)=$ $\theta_{0} \exp \left[\left(-t / \tau_{\mathrm{cr}}\right)\right]$, where $\tau_{\mathrm{cr}}$ is the creep torsional relaxation time of the visco-elastic material and $\theta_{0}$ is a constant. The typical experimental response for the RRAS thread shown in Fig. 4(a) gives a value $\tau_{\mathrm{cr}}=1 \mathrm{~min} 15 \mathrm{~s}$ in our experimental conditions. The agreement between the expected form of the responses of the silk and the observed spider dragline relaxation behavior reinforce the proposed nested model in torsion. To the best of our knowledge, this approach leads to the first observation of the torsional relaxation constants of the coherent deformations of fibroin type proteins mediated by the breaking and reform of noncovalent bonds.

Clearly, beyond their outstanding tensile, strength, and toughness qualities, spider safety draglines present us with unrivalled torsional qualities, based largely on the selfreform shape memory of the thread structure within the framework of a nested model. Besides, torsion is a powerful tool to directly isolate the fibroin type protein relaxation times. Since it has been suggested that the spider silk could be a valuable model system for exploring fibrogenesis, prions, and other insoluble related proteins [27,28], one may guess that the torsion pendulum might be a welladapted tool for investigating such domains. Such studies might also, in time, allow us to unravel the torsional dynamics of the friction and the tiny torque effects in other biological molecules.

We would like to thank B. Madsen for taking the SEM dragline picture, G. Fievet and J.-Y. Robert for providing us with Nephila draglines, C. Hamel, L. Frein, C. Deroubaix, and A. Carré for technical assistance, and J. Mevel, A. Amon, and A. Canard for early discussions.
*Corresponding author.

Electronic address: 1pl@univ-rennes1.fr

[1] C. L. Craig, Spiderwebs and Silk (Oxford University Press, New York, 2003).

[2] J. M. Gosline, M. W. Denny, and M.E. DeMont, Nature (London) 309, 551 (1984).

[3] H.-J. Jin and D. L. Kaplan, Nature (London) 424, 1057 (2003).

[4] D. Gersappe, Phys. Rev. Lett. 89, 058301 (2002).

[5] R. S. Craxton et al., Phys. Plasmas 12, 056304 (2005).

[6] F. Vollrath and D. Porter, Soft Matter 2, 377 (2006).

[7] O. Emile, A. Le Floch, and F. Vollrath, Nature (London) 440, 621 (2006).

[8] S. Zschokke, Nature (London) 424, 636 (2003).

[9] J.D. Van Beek et al., Proc. Natl. Acad. Sci. U.S.A. 99, 10266 (2002).

[10] A. Lazaris, S. Arcidiacono, Y. Huang, J.-F. Zhou, F. Dugay, N. Chretien, E. A. Welsh, J. W. Soares, and C. N. Karatzas, Science 295, 472 (2002).

[11] J. Yang, L. A. Barr, S. R. Fahnestock, and Z.-B. Liu, Transgenic Research 14, 313 (2005).

[12] C. A. Coulomb, in Mémoires de Coulomb, edited by A. Potier (Gauthier-Villars, Paris, 1884).

[13] H. Cavendish, Phil. Trans. R. Soc. London 88, 469 (1798).

[14] R. C. Ritter, L. I. Winkler, and G. T. Gillies, Phys. Rev. Lett. 70, 701 (1993).

[15] C. D. Hoyle, U. Schmidt, B. R. Heckel, E. G. Adelberger, J.H. Gundlach, D. J. Kapner, and H.E. Swanson, Phys. Rev. Lett. 86, 1418 (2001).

[16] T. R. Armstrong and M. P. Fitzgerald, Phys. Rev. Lett. 91, 201101 (2003).

[17] L. Bragg and J.F. Nye, Proc. R. Soc. A 190, 474 (1947).

[18] R.P. Feynman, R. B. Leighton, and M. Sands, The Feynman Lectures on Physics (Addison-Wesley, Reading, MA, 1964), Vol. 2.

[19] S. Ashley, Sci. Am. 289, 84 (2003).

[20] J. M. Zhang and G. Y. Guo, Phys. Rev. Lett. 78, 4789 (1997).

[21] H. S. Park, K. Gall, and J. A. Zimmerman, Phys. Rev. Lett. 95, 255504 (2005).

[22] A. Lendlein, H. Jiang, O. Jünger, and R. Langer, Nature (London) 434, 879 (2005).

[23] L. Pauling, R. B. Corey, and H. R. Branson, Proc. Natl. Acad. Sci. U.S.A. 37, 205 (1951).

[24] J. M. Gosline, P. A. Guerette, C.S. Ortlepp, and K. N. Savage, J. Exp. Biol. 202, 3295 (1999).

[25] N. Becker, E. Oroudjev, S. Mutz, J. P. Cleveland, P. K. Hansma, C. Y. Hayashi, D. E. Makarov, and H. G. Hansma, Nat. Mater. 2, 278 (2003).

[26] H. Zhou and Y. Zhang, Phys. Rev. Lett. 94, 028104 (2005).

[27] J. M. Kenney et al., European Journal of Biochemistry 269, 4159 (2002).

[28] R. J. Ellis and T. J. T. Pinheiro, Nature (London) 416, 483 (2002). 